\title{
Compte-rendu de l'Annual General Meeting de BWARS à Cambridge (Angleterre)
}

\section{Par Nicolas VEREECKEN * et Stuart PM ROBERTS **}

\section{Le groupe BWARS}

Depuis sa création en 1978 sous l'impulsion de l'IBRA (International Bee Research Association), le collectif BWARS (Bees, Wasps and Ants Recording Society) s'est remarquablement développé et connaît aujourd'hui un succès considérable. Fort de plus de 350 membres, BWARS regroupe des amateurs (professionnels ou non) ayant pour objectif principal de mieux cerner la distribution et l'écologie des Hyménoptères du Royaume-Uni. L'ensemble des observations accumulées sont compilées dans une base de données qui comprend pas moins de 300.000 observations à ce jour, et qui fut spécialement développée à cette fin. Ces données sont régulièrement mises à jour et sont à la disposition de tout un chacun, notamment dans le cadre de recherches portant sur la conservation, l'écologie et l'évolution des populations d'Hyménoptères Aculéates et de leurs habitats à l'échelle nationale.

Les membres de BWARS reçoivent également un bulletin, la BWARS Newsletter, publiée deux fois par an, ainsi que des Atlas provisoires des Hyménoptères de Grande-Bretagne et d'Irlande, publiés tous les deux ans avec le concours de la fondation BRG (Biological Records Centre). Le bulletin de BWARS propose un contenu très varié : communications brèves, cartes provisoires et fiches descriptives d'espèces, observations et informations d'intérêt pour la communauté ou relatives aux rencontres annuelles ou autres symposiums enrichissent les nombreuses pages du bulletin.

\section{Rencontre annuelle à Cambridge (UK)}

La rencontre annuelle du groupe BWARS s'est tenue durant le week-end du 30 septembre au $1^{\text {er }}$ octobre 2006 dans le cadre prestigieux du département et du musée de Zoologie de l'Université de Cambridge (Angleterre). De nombreux membres ainsi que des correspondants étrangers ont une fois de plus répondu à l'appel, contribuant ainsi au succès de cette rencontre annuelle.

La première journée (samedi 30.IX.2006), organisée dans le musée de Zoologie (Figure 1), était consacrée à

\footnotetext{
* Service d'Eco-Ethologie Evolutive, Université Libre de Bruxelles CP 160/12, Av. F.D. Roosevelt 50, B-1050 Bruxelles, Belgique.

E-mail: nicolas.vereecken@ulb.ac.be

** Centre for Agri-Environmental Research, School of Agriculture,

Policy and Development, University of Reading, Earley Gate, PO Box 237, Reading RG6 6AR, United Kingdom.

E-mail: s.p.m.roberts@reading.ac.uk
}

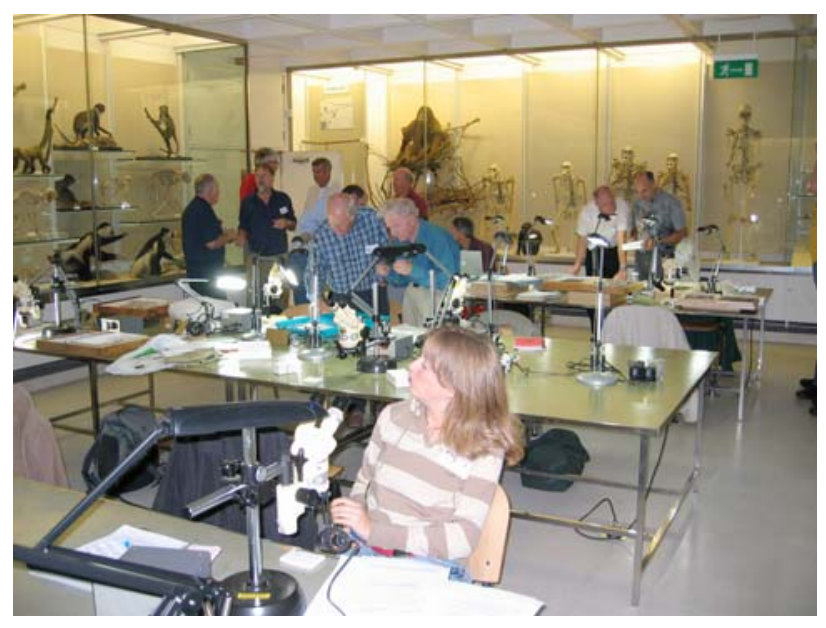

Figure 1. Séance d'identification d'insectes (Apoïdes et guêpes) au musée de Zoologie de l'Université de Cambridge (Angleterre), 30.IX.2006 (Photo N. Jones)

l'identification d'insectes sous binoculaire. Les organisateurs mirent à disposition des participants des boîtes de spécimens de référence ainsi que des clés d'identification fournies sur CD-ROM. De plus, la présence d'experts internationaux comme $\underline{\text { Paul }}$ Westrich (Kusterdingen, Allemagne), auteur du Wildbienen Baden-Württembergs (Ulmer, 1989), Michael Archer (York, Angleterre), entomologiste de renom spécialisé dans les Vespidae, ainsi que de nombreux experts anglais a considérablement stimulé les discussions tout en aidant les participants à approfondir leurs connaissances et en leur facilitant la compréhension des caractères importants utilisés dans les clés d'identification.

La seconde journée (dimanche 1.X.2006), quant à elle, était organisée au sein du département de Zoologie. L'accent fut particulièrement mis sur les communications orales, l'occasion pour les participants de découvrir les résultats des recherches menées par Jeremy Field (Londres, Angleterre), Koos Biesmeijer (Leeds, Angleterre) et Paul Westrich (Kusterdingen, Allemagne). Nigel Jones (Shrewsbury, Angleterre) a entrepris un "relooking" du site web de BWARS, qui a déjà attiré plus de 40.000 visiteurs depuis sa création, et qui devrait prochainement comporter une galerie photo ainsi que davantage de liens.

Le comité de BWARS a décidé de proposer un nouveau lieu de rendez-vous pour l'édition 2007 de la rencontre annuelle des membres du groupe. 